\title{
Comparison of Muscle Activities of the Posterior Oblique Sling Muscles among Three Prone Hip Extension Exercises with and without Contraction of the Latissimus dorsi
}

\author{
Dae-kwan Kang $\cdot$ Young-In Hwang ${ }^{\dagger}$
}

Department of Physical Therapy, College of Life and Health Science, Hoseo University

Received: May 16, 2019 / Revised: May 22, 2019 / Accepted: May 31, 2019

(C) 2019 J Korean Soc Phys Med

\section{| Abstract |}

PURPOSE: We investigated the most effective way to activate the posterior oblique sling muscles by performing prone hip extension exercises.

METHODS: An electromyography system was used to measure the activation of the posterior oblique sling muscles (latissimus dorsi, gluteus maximus, multifidus, and biceps femoris) in three different prone hip extension exercises of in 12 healthy individuals (6 men and 6 women): 1) prone hip extension, 2) prone hip extension with internal rotation and extension of the arm, and 3) prone hip extension with internal rotation and extension of the arm with a 1-Ib dumbbell.

RESULTS: The overall muscular activation of the posterior oblique sling muscles was more increased when performing 1) prone hip extension with internal rotation and 2) prone hip extension with internal rotation and extension of the arm with a 1-Ib dumbbell as compared with that during prone hip extension except for the biceps femoris activation. There was a statistically significant difference in the activities of the

†Corresponding Author : Young-In Hwang olivetree1223@gmail.com, http://orcid.org/0000-0002-7314-1678 This is an Open Access article distributed under the terms of the Creative Commons Attribution Non-Commercial License (http://creativecommons.org/licenses/by-nc/3.0) which permits unrestricted non-commercial use, distribution, and reproduction in any medium, provided the original work is properly cited. contralateral multifidi among all three exercises; of the ipsilateral multifidi in PHE1) prone hip extension alone, PHE2) prone hip extension with internal rotation and extension of the arm and PHE3) prone hip extension with internal rotation and extension of the arm with 1-Ib dumbbell; and of the ipsilateral gluteus maximus among all the prone hip extension exercises. There was no significant difference in the activity of the biceps femoris among the three exercises. CONCLUSION: Prone hip extension with internal rotation and with internal rotation and extension of the arm with 1-Ib dumbbell can activate the posterior oblique sling muscles and so prevent back pain in healthy people.

Key Words: Fasciae, Multifidus, Muscle activity, Muscle sling

\section{Introduction}

Muscular chains are groups of muscles that work together or affect simultaneously movement patterns [1]. There are synergists, muscle slings and myofascial chains, each of which has an interdependent relationship with joints or nervous tissue [1]. Muscle Slings provide stability while participating in the movement of various joints [2]. Among these muscle slings, the posterior oblique sling muscles (POSMs) comprise the opposite latissimus dorsi and gluteus 
maximus, which are connected through the thoracolumbar fascia, erector spinae, multifidus and biceps femoris, which transmit force from the lower part of the body and extend the body during gait [1-3]. Thus, the POSMs contribute to the stability of the dynamic lumbar pelvis [4].

Based on the concepts of Bergmark [5], the latissimus dorsi and erector spinae are global muscles, and the multifidus, which is also a POSM, is classified as a local muscle. The global system changes the line of action out of external forces as a prime mover, whereas the local system alters the posture of the lumbar spine to increase stability [5]. For example, the erector spinae is located outside of the trunk muscles and generates force, whereas the multifidus provides stability to the body [6]. Controlled synergy between the two muscle systems is required not only to maintain the local and global muscle systems for spinal stability (Marshall and Murphy, 2005), but also to increase spinal movement [5].

The gluteus maximus is aligned vertically to the sacroiliac joint, and so its contraction provides the transmission of force from the lower extremity to the pelvis $[7,8]$. Patients with lower back pain have a delayed onset of gluteus maximus activity and an earlier onset of biceps femoris activity [7,9].

Based on the evidence, it would be possible to prevent back pain by effectively activating the controlled synergy in the simultaneous activation of the POSMs. In addition, body movement involves several muscles to be activated while being connected with fasciae [1] for strengthening POSM to help in improving spinal mobility and stability. Vleeming et al. [10] also suggested the combined action of the POSMs for stabilizing the lumbopelvic joint. For simultaneous activation of the POSMs, several researchers have suggested prone hip extension (PHE) exercise $[2,3,11,12]$. However, there are only a few studies on increasing POSM activation during PHE according to the activation of the latissimus dorsi, which is broadly covered by the thoracolumbar fascia among the POSMs. Thus, this
Table 1. General Characteristics of the Subjects

\begin{tabular}{cc}
\hline & Mean \pm SD \\
\hline Age (years) & $23 \pm 3$ \\
Height $(\mathrm{cm})$ & $170.058 \pm 4.521$ \\
Weight $(\mathrm{kg})$ & $61.356 \pm 6.780$ \\
\hline
\end{tabular}

study investigated the most effective method of activating the POSM among three PHE exercises (prone hip extension [PHE1], prone hip extension with internal rotation and extension of the arm [PHE2], and prone hip extension with internal rotation and prone hip extension with internal rotation and extension of the arm with 1-Ib dumbbell [PHE3].

\section{Methods}

\section{Subjects}

Twelve healthy subjects participated in the study (6 men and 6 women; mean age, $23 \pm 3$ years, mean height, 170.05 $\pm 4.52 \mathrm{~cm}$; mean weight, $61.35 \pm 6.78 \mathrm{~kg}$ ). The participants in the study signed voluntary consent forms before the experiment. All the subjects used the dominant right foot and the left arm [13]. The inclusion criteria were as follows: no orthopedic and neurological disorders in the lower limbs and lumbar spine, no back pain for the past 6 months, and no history of surgery in the lumbar region and lower extremity. Patients who underwent surgery within the previous 6 months or those who had less than $10^{\circ}$ of hip and shoulder extension in the prone position were excluded from the study.

\section{Measurement}

The signals were digitally processed using a wireless electromyography (EMG) system (Wireless EMG system 1000; BTS, Italy). From the right biceps femoris to the opposite direction of the latissimus dorsi, the muscle activity of each POSM (latissimus dorsi, erector spinae, multifidus, gluteus maximus, and biceps femoris) was 

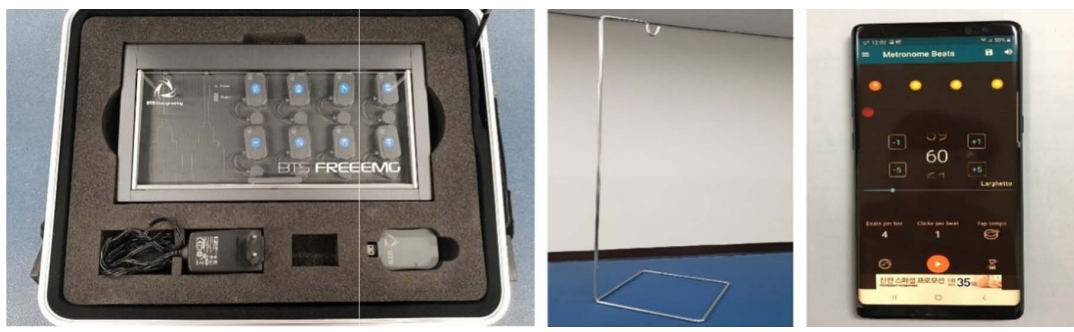

Figure 1. Methods of measurement: (a) electromyogram

(b) target bar, and (c) metronome beats

measured in the three PHE exercises. To measure each corresponding range of motion of the arm, a target bar was set at a height corresponding to $10^{\circ}$ by using a goniometer, and a metronome (Metronome Beats Pro [4.0.0]; Stonekick, UK) was used to check the performance time (Fig. 1)

\section{Procedure}

\section{1) EMG attachment}

To minimize skin resistance to the signal, the surface electrode was rubbed 3 to 4 times with thin sandpaper and alcohol-soaked cotton to remove the skin stratum. The surface electrode was attached parallel to the direction of the muscle fibers, and the distance between the electrodes was set to $2 \mathrm{~cm}$. The positions of the electrode attachments were as follows: the L2 spinous process; multifidus, $2 \mathrm{~cm}$ away from the L5 spinous process across the posterior superior iliac spine; gluteus maximus, at the center of the line connecting S4 and S5 and the greater trochanter with diagonal lines; biceps femoris, $2 \mathrm{~cm}$ from the outer surface of the leg and at two-third of the line connecting the large protrusions from the back of the knee [14](Fig. 2).

\section{2) Experimental procedure}

For PHE1, in prone position, the right lower extremity was completely extended to the knee and the hip joints were elevated to $10^{\circ}$ (Fig. 3); PHE2 is similar to PHE1, but with internal rotation and $10^{\circ}$ extension of the opposite

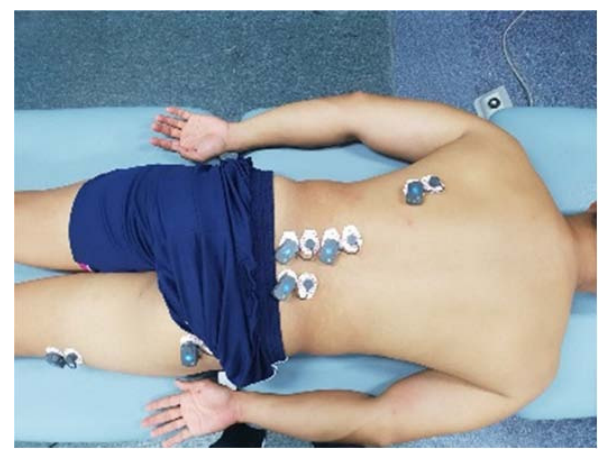

Figure 2. Electromyogram attachment

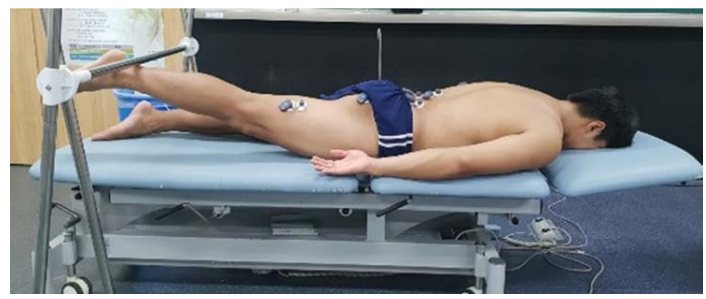

Figure 3. Prone hip extension (PHE1)

arm (Fig. 4). PHE3 is similar to PHE2, but a 1-Ib dumbbell is added (Fig. 5). The target bar was set up so that each subject held the arm and leg up to the target bar corresponding to $10^{\circ}$, and the investigator provided continuous feedback to the subject. Before the experiment, maximal voluntary isometric contraction (MVIC) was measured, and a 2-min rest period was provided after each test for the three PHE exercises. Each test was performed for $5 \mathrm{sec}$, and a rest period of $3 \mathrm{sec}$ was provided between repetitions. The raw data were collected for RMS 


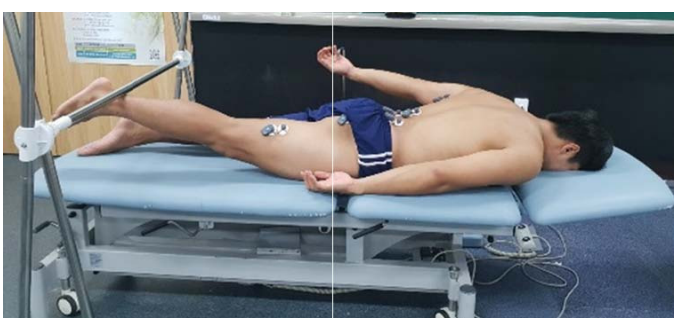

Figure 4. Prone hip extension with internal rotation and extension of the arm (PHE2)

processing after 3 repetitions, and the mean value was analyzed as \%MVIC.

\section{Data analysis}

The SPSS program version 12.0 was used for the statistical analysis. One-way repeated measures ANOVA was used to determine the percentage of activity of the POSM in the three different PHE exercises. Bonferroni corrections were conducted as post hoc tests. The level of statistical significance was set at .017 .

\section{Results}

The POSM activities were significantly increased in the PHE1, PHE2, and PHE3 exercises. In particular, a significant difference was found in the activity of the left latissimus dorsi in PHE1, PHE2, and PHE3 and in the activity of the left eretor spinae in PHE1 and PHE3. There was a statistically significant difference in the activities of the left posterior multifidus in PHE1, PHE2, and PHE3, in the right multifidus in PHE1 and PHE3, and in the right gluteus maximus in all PHE exercises (Table 2). There was no significant difference during the activity of the biceps femoris during all the PHE exercises (Table 2).

\section{Discussion}

This study was conducted to investigate the PHE

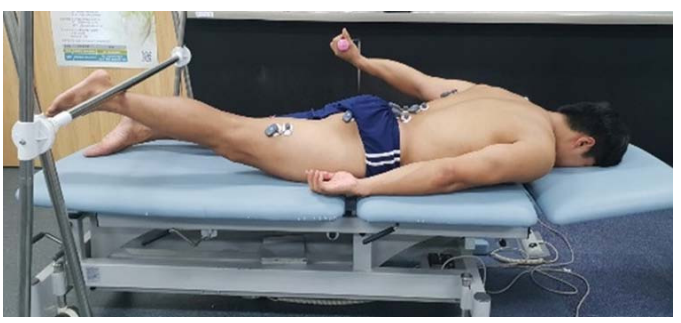

Figure 5. Prone hip extension with internal rotation and extension of the arm with 1-lb dumbbell (PHE3)

position that caused the most effective POSMs activation. The results showed that the activities of POSMs, except for the biceps femoris, were significantly increased at PHE3 $(p<.017)$ (Table 2). There were significant differences in the activities of the left latissimus dorsi, the erector spinae, the multifidus $(\mathrm{p}<.017)$, the right multifidus and gluteus maximus $(p<.017)$. However, there was no significant difference in the biceps femoris during all PHE exercises $(\mathrm{p}>$.017).

The activities of the POSMs, except for that in the biceps femoris, were increased in the PHE3, which would be due to the co-contraction of the latissimus dorsi with extra load (the 1-Ib dumbbell) with involvement of the thoracolumbar fasciae. In particular, the right gluteus maximus muscle activity was significantly increased in PHE3 compared to that in PHE1 because the latissimus dorsi muscle activity increases to maintain the external load during contralateral shoulder extension and internal rotation, and this could act as a global muscle to stabilize the trunk [1]. The increase in activity of both multifidus muscles is the result of increased stabilization of the spinal segments when spine movement occurs due to the simultaneous contraction of the local and global muscles.

The activity of the biceps femoris was gradually inhibited in PHE2 and PHE3, but no significant difference was found between the three PHE exercises. The reason for this was that the PHE position influenced the activity of the biceps femoris based on the study by Kang [12] 
Table 2. Electromyohraphic Activities of the Various Muscles

\begin{tabular}{|c|c|c|c|c|c|}
\hline \multirow{2}{*}{ Muscle } & \multicolumn{3}{|c|}{ Mean \pm SD } & \multirow{2}{*}{$\mathrm{F}$} & \multirow{2}{*}{$\mathrm{p}$} \\
\hline & apHE1 & bPHE2 & 'PHE3 & & \\
\hline${ }^{\mathrm{d}} \mathrm{LD}(\mathrm{Lt})$ & $24.172 \pm 18.567$ & $50.177 \pm 43.862$ & $62.145 \pm 49.853$ & 8.714 & $.002^{*}$ \\
\hline${ }^{\mathrm{e}} \mathrm{ES}$ (Lt) & $30.335 \pm 11.539$ & $35.912 \pm 11.644$ & $39.221 \pm 11.082$ & 8.517 & $.002^{*}$ \\
\hline${ }^{\mathrm{f}} \mathrm{MF}(\mathrm{Lt})$ & $48.788 \pm 24.567$ & $57.138 \pm 26.572$ & $59.604 \pm 23.330$ & 12.070 & $.000^{*}$ \\
\hline${ }^{\mathrm{g}} \mathrm{MF}$ (Rt) & $45.087 \pm 19.850$ & $49.416 \pm 22.367$ & $52.833 \pm 20.727$ & 6.106 & $.010^{*}$ \\
\hline${ }^{\mathrm{h}} \mathrm{GM}$ (Rt) & $22.380 \pm 13.647$ & $30.022 \pm 19.244$ & $35.615 \pm 18.098$ & 21.570 & $.000^{*}$ \\
\hline${ }^{\mathrm{i}} \mathrm{BF}$ (Rt) & $46.273 \pm 20.502$ & $48.670 \pm 30.633$ & $43.637 \pm 21.809$ & 1.203 & .319 \\
\hline
\end{tabular}

${ }^{\mathrm{a}} \mathrm{PHE}$ 1: Prone hip extension.

${ }^{b}$ PHE 2: Prone hip extension with the arm in internal rotation and extension.

${ }^{c}$ PHE 3: Prone hip extension with the arm in internal rotation and extension with a 1-lb dumbbell.

${ }^{\mathrm{d}} \mathrm{LD}(\mathrm{Lt})$ : Latissimus dorsi on the left side.

${ }^{e} \mathrm{ES}(\mathrm{Lt})$ : Erector spinae on the left side.

${ }^{\mathrm{f}} \mathrm{MF}$ (Lt): Multifidus on the left side.

${ }^{\mathrm{g}} \mathrm{MF}$ (Rt): Multifidus on the right side.

${ }^{\mathrm{h}} \mathrm{GM}(\mathrm{Rt})$ : Gluteus maximus on the right side.

${ }^{\mathrm{i}} \mathrm{BF}(\mathrm{Rt})$ : Biceps femoris on the right side.

It is presumed that in that study, measurement was not obtained in the hip joint abduction posture, but rather, measurement was done in the extension position.

Currently, there are numerous studies on PHE positions that could selectively strengthen the gluteus maximus [15]. In addition, when lower abdominal hollowing was applied simultaneously in the prone position of the hip, the onset time of the gluteus maximus activity was less likely to be delayed, but it was not statistically significant [13]. On comparing the EMG of the POSM before and after pre-activation of the lower trapezius, the activation of the ipsilateral gluteus maximus and bilateral multifidus was significantly greater during the pre-activation of the lower trapezius [2]. One of the reasons for this is that pelvic rotation and anterior tilting are increased in those cases where lower abdominal hollowing is not simultaneously applied [2].

Furthermore, Kim et al. [3] showed significant improvement in the contralateral latissimus dorsi, the ipsilateral erector spinae, and gluteus maximus activities when these were measured by POSM activation on an unstable support and when compared with that in a stable support. This suggests that the thoracolumbar fascia significantly contributes to the stability of the hip and pelvis, which is similar to the results of this study. It has been shown that the activity of the latissimus dorsi may have an effect on the activation of the gluteus maximus since the POSM is linked to the thoracolumbar fasciae [1]. Therefore, co-activation of the latissimus dorsi with extraloading at the PHE position (PHE3) activates the erector spinae, multifidus, and gluteus maximus connected to the POSM, resulting in a more clinically effective method.

The limitation of this study is that it is difficult to apply this method to patients with certain conditions, such as back pain, because this study recruited only healthy subjects. People with back pain have a different pattern of trunk muscle coordination and co-contraction of the back [16] or muscle activities [17]. In the future, this type of study should be applied to patients with low back pain and our finding can be used to prevent low back pain in 
healthy people.

\section{Conclusion}

The purpose of this study was to investigate the most effective ways to activate the POSMs in three different prone hip extension exercises. The activities of the POSMs (latissimus dorsi, gluteus maximus, multifidus, biceps femoris) in three different PHE exercises (PHE1, PHE2, and PHE3) were compared. As a result, in PHE3, the activities of the POSMs, except the biceps femoris, were significantly increased. Therefore, the PHE3 exercise is recommended to be used in clinical settings, and it could simultaneously improve the activities of the POSMs as a preventive exercise for healthy subjects. However, the effect of the activities of POSMs in the three different PHE exercises for patients with back pain should be investigated in the future.

\section{References}

[1] Myers TM. Anatomy trains: Myofascial meridians for manual and movement therapists. 3rd ed. Edinburg, Churchill Livingstone, 2014.

[2] Jeon IC, Ha SM, Hwang UJ, et al. Comparison of EMG activity of the posterior oblique sling muscles and pelvic rotation during prone hip extension with and without lower trapezius pre-activation. Physical Therapy Korea. 2016;23(1):80-6.

[3] Kim JW, Han JY, Kang MH, et al. Comparison of Posterior Oblique Sling Activity during Hip Extension in the Prone Position on the Floor and on a Round Foam Roll. J Phys Ther Sci. 2013;25(8):977-9.

[4] Oh YC, Cynn HS, Yi CH, et al. Effect of hip external rotation angle on pelvis and lower limb muscle activity during prone hip extension. Physical Therapy Korea. 2014;21(3):1-10.

[5] Bergmark A. Stability of the lumbar spine. A study in mechanical engineering. Acta Otthop Scand Suppl. 1989; 230:1-54.

[6] Panjabi MM. The stabilizing system of the spine. part I. function, dysfunction, adaptation, and enhancement. J Spinal Disord. 1992;5(4):383-9.

[7] Hossain M, Nokes LD. A model of dynamic sacro-iliac joint instability from malrecruitment of gluteus maximus and biceps femoris muscles resulting in low back pain Med Hypotheses. 2005;65(2):278-81.

[8] Lieberman DE, Raichlen DA, Pontzer H, et al. The human gluteus maximus and its role in running. J Exp Biol. 2006;209(11):2143-55.

[9] Hungerford B, Gilleard W, Hodges P. Evidence of altered lumbopelvic muscle recruitment in the presence of sacroiliac joint pain. Spine (Phila Pa 1976). 2003;28(14): 1593-600.

[10] Vleeming A, Pool-Goudzwaard AL, Stoeckart R, et al. The posterior layer of the thoracolumbar fascia. Its function in load transfer from spine to legs. Spine (Phila Pa 1976) 1995;20(7):753-8.

[11] Lee JG. The effects of gluteus maximus contraction levels on posterior oblique sling muscles activations during prone hip extension exercise on prone position. Daegu, Catholic University of Daegu, Master dissertation. 2016.

[12] Kang SY, Jeon HS, Kwon O, et al. Activation of the gluteus maximus and hamstring muscles during prone hip extension with knee flexion in three hip abduction positions. Manual Therapy. 2013;18(4):303-7.

[13] Chance-Larsen K, Littlewood C, Garth A. Prone hip extension with lower abdominal hollowing improves the relative timing of gluteus maximus activation in relation to biceps femoris. Manual Therapy. 2010;15(1):61-5.

[14] Cram JR, Kasman GS, Holtz J. Introduction to surface electromyography. London, Jones and Barlett Publishers. 1998.

[15] Kisner C, Colby LA. Therapeutic exercise: foundations and technique. 5th ed. Philadelphia, PA, F. A. Davis Co. 2007. 
[16] D'hooge R, Hodges P, Taso H, et al. Altered trunk muscle coordination during rapid trunk flexion in people in remission of recurrent low back pain. J Electromyogr kinesiol. 2013;23(1):173-81.

[17] Larivière C, Gagnon D, Loisel P. The comparison of trunk muscles EMG activation between subjects with and without chronic low back pain during flexionextension and lateral bending tasks. J Electromyogr Kinesiol. 2000;10(2):79-91. 